\title{
Sleep EEG characteristics associated with total sleep time misperception in young adults
}

\section{Biyun Xu ( $\nabla$ cloudxby@163.com )}

Second Affiliated hospital of Guangzhou University of chinese medicine https://orcid.org/0000-00020422-885X

\section{Qinghao Cai}

Guangdong Provincial Hospital of Traditional Chinese Medicine

\section{Runru Mai}

Guangdong Provincial Hospital of Traditional Chinese Medicine

Hailong Liang

Guangdong Provincial Hospital of Traditional Chinese Medicine

Jiayu Huang

Guangdong Provincial Hospital of Traditional Chinese Medicine

\section{Zhimin Yang}

Guangdong Provincial Hospital of Traditional Chinese Medicine

\section{Research}

Keywords: adult, misperception, EEG, power spectral analysis, cortical activation

Posted Date: March 26th, 2021

DOI: https://doi.org/10.21203/rs.3.rs-332688/v1

License: (c) (i) This work is licensed under a Creative Commons Attribution 4.0 International License.

Read Full License 
Sleep EEG characteristics associated with total sleep time misperception in young adults

Biyun $\mathrm{Xu}^{1,2 \#}$, Qinghao Cai ${ }^{2}$, Runru $\mathrm{Mai}^{2}$, Hailong Liang ${ }^{2}$, Jiayu Huang ${ }^{2}$, Zhimin Yang ${ }^{2}$.

${ }^{1}$ Applicants for doctoral degree with an equivalent educational level in Guangzhou University of traditional Chinese Medicine, Guangzhou, 510720, China.

${ }^{2}$ Department of Sleep-disorder, Fangcun Branch, The Second Affiliated Hospital of Guangzhou University of Chinese Medicine (Guangdong Provincial Hospital of Chinese Medicine), Guangzhou, 510370, China.

\section{\#Corresponding author}

Biyun Xu

Applicants for doctoral degree with an equivalent educational level in Guangzhou University of traditional Chinese Medicine

36 Yong'an street, Liwan District, Guangzhou, 510370, China

cloudxby@163.com

Tel:13632399892 
Sleep EEG characteristics associated with total sleep time misperception in young adults

Backgroud: Power spectral analysis (PSA) is one of the most commonly-used EEG markers of cortical hyperarousal which help to understand subjective-objective sleep discrepancy (SOD). Age is associated with decreased sleep EEG activity. Currently, PSA of young adults are limited. Thus, this study aimed to examine the correlation of spectral EEG power with total sleep time misperception in young patients.

Methods: Forty-seven young adults were recruited and underwent a polysomnography recording in a sleep laboratory. The clinical records and self-report questionnaires of all patients were collected, who were categorized into the GS $(n=10)$, insomnias with a low mismatch (IWLM, $\mathrm{n}=19$ ) and participants with a high mismatch (IWHM, $\mathrm{n}=18$ ) subgroups. Power spectral analysis was performed during the first 6 hours of sleep.

Results: The IWLM group showed increased absolute beta power in central-frontal area and relative beta power in frontal areas compared to the GS group. In addition, IWHM patients exhibited higher absolute and relative beta power in the central area compared to the GS group. The absolute and relative beta/delta ratios in frontal area in the IWHM and IWLM groups were higher than those in the GS group. The IWHM group also showed higher absolute and relative beta/delta ratios in the central area compared with the GS group. No significant difference in the above parameters was observed between the IWHM and IWLM groups. Moreover, the SOD of TST was negatively correlated with the relative delta power $(\mathrm{r}=0.289, \mathrm{p}=0.049)$, beta power $(\mathrm{r}=0.373, \mathrm{p}=0.010)$, beta/delta ratio $(\mathrm{r}=0.314, \mathrm{p}=0.032)$, and the absolute beta/delta ratio $(\mathrm{r}=0.314, \mathrm{p}=0.032)$ in central area. 
Conclusions: Young IWHM and IWLM patients showed increased beta EEG power compared to GS, suggesting that there exists increased cortical activity in these patients. Also, the beta/delta ratio was negatively correlated with the SOD in patients with IWHM and IWLM.

Keywords: young adult; misperception; EEG; power spectral analysis; cortical activation 
Sleep EEG characteristics associated with total sleep time misperception in young adults

\subsection{Introduction}

Insomnia is a common disease in modern society with a prevalence rate ranging from $12-20 \%{ }^{1}$. Patients with insomnia have an increased risk of developing mental illness $^{2}$ and physical diseases ${ }^{3,4}$, which is often accompanied by increased healthcare and medical costs, higher absenteeism, higher incidence of traffic accidents and falls, and a reduced quality of life ${ }^{4-7}$.

Subjective-objective sleep discrepancy (SOD) refers to the underestimation of total sleep time (TST) and overestimation of sleep onset latency (SOL), and is very ubiquitous in insomnia patients ${ }^{8,9}$. It could reach an extreme degree in Paradoxical insomnia (Par-I), which was used to be named in the International Classification of Sleep Disorders - 2rd Edition (ICSD-2), but cancelled in ICSD -3 mainly due to lack of agreement on its precise definition. Although the disease name has been cancelled, the phenomenon it represents is common in insomnia patients. It is worth to study ${ }^{10}$, and is an important aspect to understand insomnia.

Several researches have been studied to explain the SOD in insomnias referring thirteen possible mechanisms supported by good-quality evidence ${ }^{8}$.One of them is about cortical hyperarousal. Power spectral analysis (PSA) is one of the most commonly-used EEG markers of cortical hyperarousal ${ }^{11}$ and it represents enhanced sensory information processing, which can lead to sleep state misperception ${ }^{12}$. The power of each waveform is defined as the area below the waveform with the greater the amplitude indicating greater power ${ }^{8}$. 
Previous studies have shown that high-frequency EEG activity, especially beta activity, is increased in patients with primary insomnia (PI) at sleep onset, during nonrapid eye movement (NREM) sleep ${ }^{13-16}$, and even during wakefulness ${ }^{17,18}$. This divergence may be explained by one of the fact that the types of insomnia are not considered in most studies, for example ${ }^{8}$, individuals with and without misperception. Krystal et al. ${ }^{19}$, St-Jean et $a .^{20}{ }^{20}$ and Sandro Lecci et al. ${ }^{21}$ categorized their patients according to different types of insomnia. However, there was no consistency in the spectral power and only elderly patients (40-80 years old vs. $40.21+9.38$ vs $40-85$ years old) were included in these studies. It has also been shown that age is associated with decreased sleep EEG activity ${ }^{22,23}$. Less slow-wave and spindle activity during non-rapid eye movement (NREM) sleep, together with attenuated levels of rapid eye movement (REM) sleep are exhibited in older people than that in young individuals ${ }^{24}$. In this study, we aimed to compare the TST in young patients who overestimated their sleep by at least two hours, relative to a group who correctly estimated their sleep, and good sleepers (GS). Our findings may be important for clinical and public health and for the treatment and management of insomnia ${ }^{8}$.

\subsection{Materials and Methods}

\section{Participants}

Seventy participants aged between 18 to 40 years were recruited from the Guangdong Provincial Hospital of Chinese Medicine through posters from May 2016 to November 2017. All subjects were asked to complete a two-week sleep diary followed by a single all-night PSG recording in a sleep monitoring room. Personal information was obtained from all subjects and included age, sex, race, place of residence, marital status, family history of insomnia, and family history of psychosis. Two self-reported 
questionnaires, the Pittsburgh Sleep Quality Index (PSQI) ${ }^{25}$ and the Symptom Checklist 90 (SCL-90), were given to each participant. Subjective sleep quality was determined by self-reported TST after PSG. The following questions were asked to the subjects within two hours after completion of polysomnography using standardized questions: "How long did you sleep last night?" and "Did you sleep as usual?"

Subjects were eligible to be enrolled as GS if they 1) reported no difficulty in sleep according to the two-week sleep diary (i.e. a SOL of $<30 \mathrm{~min}$, awakenings of $<40$ min, a TST between 6.0 to $8.0 \mathrm{~h}$, or a sleep efficiency (SE) of $\geq 85 \%$ ); 2) had a PSQI score of $<7^{25}$. Participants with a SE of $<85 \%$ or sleep time of $<6$ h were excluded from this study.

Participants were defined as insomnia patients if they 1) met the diagnostic criteria for chronic insomnia disorder (International Classification of Sleep Disorders, $3^{\text {rd }}$ edition); 2) reported at least three nights per week of sleep difficulty (i.e. a SOL of $>30 \mathrm{~min}$, awakenings of $>40 \mathrm{~min}$, a TST of $<6.0 \mathrm{~h}$, or a SE of $<85 \%$ ); 3) had a PSQI score of $>7$; 4) had difficulty sleeping for more than three months; 5) did not have other medical, psychological, or sleep disorders or take other medications.

Insomnia patients were further categorized into the insomnias with a low mismatch (IWLM) and participants with a high mismatch (IWHM) subgroups. IWLM were defined as individuals who met the criteria for chronic insomnia disorder and had a SOD of $<60$ min in TST. IWHM were defined as those who met criteria for chronic insomnia disorder and had normal PSG parameters (i.e. an SE of $>85 \%$ and a TST of $>$ $6.5 \mathrm{~h}$ ) and a SOD of $>120 \mathrm{~min}$ in TST. In both the IWHM and IWLM groups, patients 
were excluded if they 1) were diagnosed with another Axis I disorder or any other sleep disorders(e.g. idiopathic insomnia, sleep apnea, which was defined as an apneahypopnea index of more than five events per hour using PSG, or restless leg syndrome); 2) were affected by other external factors that might affect insomnia (e.g., physical pain caused by medical diseases, drugs affecting sleep structure, alcohol consumption, other treatments, etc.); 3) went to sleep later than 0:00am or woke up before 6:00 am, or had irregular sleep schedules.

Based on the inclusion and exclusion criteria, 47 participants were included in the study: GS group ( $\mathrm{n}=10 ; 5$ males,5 females), IWLM group ( $\mathrm{n}=19 ; 3$ males, 16 females), and IWHM group ( $\mathrm{n}=18 ; 9$ males, 9 females).

\section{PSQI and SCL-90}

The PSQI is a questionnaire consisting of 21 items and has been commonly used to evaluate subjective sleep quality. The higher the score, the greater the severity of insomnia. A score of $>7$ indicates abnormal sleep (severe difficulty in at least two areas or moderate difficulty in more than three areas).

The SCL-90 is one of the most widely used mental health scales in the field of psychiatry. It is a 90-item, self-reported symptom inventory. The score for each item is summed, yielding a total score that covers ten aspects. The higher the total score, the greater the risk of developing psychological distress ${ }^{27}$. 


\section{PSG Recordings}

In conventional PSG (Nicolet, ONE, EEG 32, USA), the international 10-20 system is used to record EEG. In the current work, the grounding electrode was placed on the frontal pole midline point and the bilateral ear electrodes were used as the reference. An electrographic electrode was placed near each eye to track eye movement. The impedance was kept below $5 \mathrm{k} \Omega$ for all electrodes. The surface electrodes included four EEG (two central electrodes (C3, C4), two frontal EEG electrodes (F3, F4)), two electrooculogram (EOG), submental electromyogram (EMG), electrocardiogram (ECG), and two reference electrodes (M1, M2). In addition, tibialis EMG and respiration were monitored to exclude artifacts due to periodic limb movements (a myoclonus arousal index of $>15$ ) and sleep apnea (an apnea-hypopnea index of $>5$ ). Participants were asked to sleep at their usual time (before 0:00am) and wake up at 7:00 am. The sampling rate for EEG acquisition was $500 \mathrm{~Hz}$ and the filter settings were as follows: notch frequency60 Hz; low pass filter $35 \mathrm{~Hz}$; high pass filter $0.3 \mathrm{~Hz}$.

Sleep records were reviewed and scored by a registered polysomnography technician according to the revised AASM 2.5 sleep scoring criteria ${ }^{28}$. The sleep continuity parameters, including TST, SPT, SE (ratio of TST to time in bed $\times 100$ ), and SOL, and sleep architecture parameters, including the number of awakenings, the number of arousals, arousal index, percentage of stages 1 and 2, slow wave sleep (SWS), and rapid eye movement (REM) sleep of TST were analyzed. 


\section{Spectral Analysis}

The time-domain data from the first 6-h of sleep from a central and frontal EEG electrode (averaged C3-A2 and C4-A1 channels, averaged F3-A2 and F4-A1 channels) were transformed into the frequency-domain using the fast Fourier transforms (FFT). EMG artifacts were automatically detected and rejected from all analysis ${ }^{29}$. EEG was amplified with a solution of $0.25 \mathrm{~Hz}$, an EEG segment length of $6 \mathrm{~h}$, a bandpass filter setting of $0.5-30 \mathrm{~Hz}(-3 \mathrm{~dB})$, and a frequency of $200 \mathrm{~Hz}$.

The beta $(13-30 \mathrm{~Hz})$, alpha $(8-13 \mathrm{~Hz})$, delta $(0.5-4 \mathrm{~Hz})$, and theta $(4-8 \mathrm{~Hz})$ band activity were extracted for PSA analysis. The values of relative spectral power were calculated by dividing the absolute power of each frequency band by the power of the total power spectrum. The values of the absolute and relative spectral power of the NREM sleep were log-transformed before data analysis.

\section{Statistical Analysis}

Statistical analysis was performed by the SPSS software (ver. 24.0) using unpaired two-tailed tests of significance. One-way ANOVAs or non-parametric analysis with post-hoc analysis was used to evaluate statistical significance between groups and family-wise error rate due to multiple comparisons was controlled using Bonferroni's correction (corrected $\alpha=0.002$ ). Levene's test and the Normal test were performed before an ANOVA to examine the homogeneity and distribution of the variance. Pearson's correlation analysis was used to determine the correlation between EEG spectral power (absolute and relative) and the SOD of TST. $P<0.05$ was considered statistically significant. 


\subsection{Results}

\subsubsection{Baseline characteristics}

There was no significant difference in age, sex, race, place of residence, marital status, family history of insomnia, or family history of psychosis among the three groups (Table 1).

\subsubsection{PSQI, SCL90, and PSG characteristics}

The comparisons of the PSQI score, SCL-90 score, and PSG among the three groups are shown in Table 2. The IWHM and IWLM groups showed higher PSQI and SCL90scores compared to the GS group. However, there was no significant difference in the PSQI or SCL-90 scores between the IWHM and IWLM groups. The PSG parameters were not significantly different among the GS, IWHM, and IWLM groups.

\subsubsection{Absolute EEG spectral power}

Post-hoc analysis (Kruskal-Wallis test) revealed that the IWLM group exhibited significantly higher beta power in the frontal area than the GS group. Also, the beta power in the central area was significantly higher in the IWHM and IWLM groups compared to the GS group. The IWHM and IWLM groups exhibited a significantly higher beta/delta ratio in the frontal area than the GS group. A significantly higher beta/delta ratio in the central area was also observed in the IWHM group compared tothe GS group. There was no significant difference in these parameters between the IWHM and IWLM groups (Table 3). 


\subsubsection{Relative EEG spectral power}

The average NREM activity for beta activity and the beta/delta ratio in the central or frontal area were significantly different among groups. Post-hoc analysis (KruskalWallis test) showed that the frontal beta power in the IWLM group was higher than that in the GS group. Also, the IWHM group had a significantly higher beta power in the central area compared to the GS group. A significantly higher beta/delta ratio in the frontal area was found in the IWHM and IWLM groups compared with the GS group. The IWHM group also had a significantly higher beta/delta ratio in the central area than the GS group. No significant difference in relative spectral power was observed between the IWHM and IWLM groups (Table 4).

\subsubsection{Correlation between absolute EEG spectral power and SOD}

The absolute EEG spectral power and beta/delta ratio in the central area were positively and significantly correlated with the SOD of TST $(\mathrm{r}=0.314, p=0.032)$ (Table $5)$.

\subsubsection{Correlation between relative EEG spectral power and SOD}

The relative delta $(\mathrm{r}=0.289, \mathrm{p}=0.049)$ and beta $(\mathrm{r}=0.373, p=0.010)$ power, as well as the beta/delta ratio $(\mathrm{r}=0.314, p=0.032)$ in the central area were significantly correlated with the SOD of TST $(\mathrm{r}=0.314, p=0.032)$ (Table 6$)$.

\subsection{Discussion}

This is the first study, to the best our knowledge, that has investigated the absolute and relative spectral power of young adult patients (18-40years old) with insomnia. Here, we categorized insomnia patients into the IWLM and IWHM groups to 
maximize the difference in the SOD. Compared to the GS group, patients with IWLM and IWHM exhibited an increase in the absolute and relative beta $(13-30 \mathrm{~Hz})$ frequencies and the beta/delta ratio in the central-frontal area during sleep. Moreover, the SOD of TST was positively correlated with the absolute and relative beta/delta ratio in the central area in insomnia patients. However, no significant difference was observed in the EEG power or beta/delta ratio in the central area between the IWLM and IWHM groups. Also, there was no significant difference in the absolute and relative beta power in the frontal area between the IWHM and GS groups.

Beta power is generally thought to be an index of cortical arousal. It has been shown that beta activity in the PI-group is higher than that in $\mathrm{GS}^{13-16}$. Here, we found that the IWHM group exhibited a significantly increased absolute and relative beta power in the central region, indicating increased cortical arousal during sleep. These results suggest that the IWHM group may experience enhanced sensory processing during sleep, which may render them highly responsive and sensitive to external sounds. This in turn may also lead to the mistaken perception of their sleep as wakefulness ${ }^{12}$. Our findings are similar to a previous EEG-based spectral investigation by Krystal et al. ${ }^{19}$ that showed greater beta NREM EEG activity in IWHM patients relative to GS. Nevertheless, unlike this previous work, we did not observe lower delta NREM EEG activity, or greater alpha, sigma (11-14 Hz), or beta NREM EEG activity in the IWHM group. This discrepancy may be attributed to the differences in the age and diagnostic criteria of IWHM patients between the two studies.

In our study, the IWLM group revealed increased absolute and relative beta power in the frontal area and increased absolute beta power in the central area compared to 
GS. These results are again inconsistent with the results reported by Krystal et al. ${ }^{19}$ and St-Jean et al. ${ }^{20}$ that showed no difference between IWLM and GS participants. One possible explanation is that patients of different ages were selected in the various studies and that different sleep stages were analyzed.

Recently, the ratio of high-frequency to low-frequency EEG power has been recognized as a novel indicator of cortical arousal. Furthermore, individuals with a higher ratio of this sort may have more sleep difficulties. Meric et al. ${ }^{30}$ found that IWLM patients exhibited an increased beta/delta ratio in the temporal lobe during the sleep onset period (SOP). However, some studies have also reported that delta EEG activity is decreased in IWLM patients in the temporal and central brain areas during the $\mathrm{SOP}^{18,31}$. Thus, such an activity index (beta/delta ratio) may be a more appropriate indicator of cortical arousal in insomnia patients ${ }^{16,30,32}$.In the current study, both the IWHM and IWLM groups showed increased absolute and relative beta/delta ratio in the central area when compared with the GS group.

We further showed that the SOD of TST in insomnia patients was moderately associated with the absolute NREM beta/delta ratio $(\mathrm{r}=-.314)$, relative beta power $(\mathrm{r}=-.373)$, and relative beta/delta ratio $(\mathrm{r}=-.314)$, and slightly associated with the relative delta spectral power $(\mathrm{r}=-.289)$ in the central area. Overall, these results indicate that the higher the beta/delta ratio and beta power during NREM sleep, the greater underestimation of TST. Our results are similar to the findings by Perlis et $a l^{13}$. which showed a moderate correlation between the SOD of TST and NREM beta activity $(14-35 \mathrm{~Hz})(\mathrm{r}=-.46)$. The underestimation of TST may be explained by the insertion of high frequency EEG into low frequency EEG, which has been shown to enhance information processing and reduce sleep quality ${ }^{33}$. 
There are various limitations to our study that must be noted. First, only one PSG recording was performed in each participant and thus, the results might be biased by the "first night" effect. Secondly, insomnia patients were categorized into the IWHM and IWLM groups based only on TST and the percentage of SWS should also be considered in future investigations. Lastly, the sample size in this work was relatively small. Future studies with larger sample sizes are needed to further elucidate the neurophysiological mechanisms about the SOD.

\subsection{Conclusion}

In conclusion, the present study suggests that insomnia in young adults is associated with increased absolute beta EEG power in the central brain area. Compared to healthy subjects, IWHM patients exhibited increased relative beta power in the central area and IWLM patients exhibited higher beta power in the frontal area, indicating that that cortical hyper arousal was present in all insomnia patients. Hyperarousal (e.g., beta EEG power) in the frontal area was only observed in patients with IWLM. Moreover, the SOD of TST was associated with beta/delta ratio, indicating sleep disruption in insomnia patients.

\section{Acknowledgments}

We want to thank Chen Wang and Yujiao Sun who helped in the statistical analysis.

\section{Author contributions}

Biyun Xu: data analysis and interpretation, manuscript drafting/revision. Qinghao Cai: major role in data acquisition. Runru Ma: manuscript revision. Hailong Liang: study supervision, critical revision of the manuscript. Jiayu Huang: data collection, 
manuscript revision. Zhimin Yang: study concept and design, critical revision of the manuscript.

\section{Funding}

This study was supported by 2014 Science and Technology Projects of Guangdong Province [2014A020212557]; Zhimin Yang's academic experience inheritance studio project of Guangdong Provine famous TCM [Document of Guangdong Provine (2018) No. 150].

\section{Availability of data and materials}

The datasets used and/or analysed during the current study are available from the corresponding author on request.

\section{Ethics approval and consent to participate}

This clinical trial was approved by the Ethics Committee of the Guangdong Provincial Hospital of Chinese Medicine (number: B3016-075) and performed in accordance with the World Medical Association Declaration of Helsinki. This study was registered on http://www.chictr.org/up (registration number: chiCTR-COC-16008530). Informed consent was obtained from all subjects prior to participation.

\section{Consent for publication}

Not applicable.

\section{Competing interests}

The authors declare that they have no competing interests. 


\section{Author details}

${ }^{1}$ Applicants for doctoral degree with an equivalent educational level in Guangzhou University of traditional Chinese Medicine, Guangzhou, 510720, China.

${ }^{2}$ Department of Sleep-disorder, Fangcun Branch, The Second Affiliated Hospital of Guangzhou University of Chinese Medicine (Guangdong Provincial Hospital of Chinese Medicine), Guangzhou, 510370, China.

\section{References:}

$\begin{array}{llll}\text { 1. Buysse } & \text { DJ. Insomnia. }\end{array}$ http://dx.doi.org/10.1001/jama.2013.193

2. Baglioni C, Battagliese G, Feige B, et al. Insomnia as a predictor of depression: A meta-analytic evaluation of longitudinal epidemiological studies. J Affect Disorders 2011;135(1-3):10-9. http://dx.doi.org/10.1016/j.jad.2011.01.011

3. Laugsand LE, Vatten LJ, Platou C, Janszky I. Insomnia and the Risk of Acute Myocardial Infarction. Circulation 2011; $124 \quad$ (19): 2073-81. http://dx.doi.org/10.1161/CIRCULATIONAHA.111.025858

4. Riemann D, Spiegelhalder K, Espie C, et al. Chronic insomnia: clinical and research challenges--an agenda. Pharmacopsychiatry 2010;44(1):1-14. http://dx.doi.org/10.1055/s-0030-1267978

5. Hillman D, Mitchell S, Streatfeild J, Burns C, Bruck D, Pezzullo L. The economic cost of inadequate sleep. Sleep 2018;41(8). http://dx.doi.org/10.1093/sleep/zsy083 
6. Sivertsen B, Lallukka T, Salo P. The Economic Burden of Insomnia at the Workplace. An Opportunity and Time for Intervention? Sleep 2011;34(9):1151-2. $\underline{\text { http://dx.doi.org/10.5665/SLEEP.1224 }}$

7. Hillman DR, Murphy AS, Pezzullo L. The economic cost of sleep disorders. Sleep 2006;29(3):299-305. http://dx.doi.org/10.1093/sleep/29.3.299

8. Harvey AG, Tang NKY. (Mis)perception of sleep in insomnia: A puzzle and a resolution. Psychol Bull 2012;138(1):77-101. http://dx.doi.org/10.1037/a0025730

9. Tang NKY, Harvey AG. Time Estimation Ability and Distorted Perception of Sleep in Insomnia. Behav Sleep Med. 2005;3(3):134-50. http://dx.doi.org/10.1207/s15402010bsm0303_2

10. Castelnovo A, Ferri R, Punjabi NM, et al. The paradox of paradoxical insomnia: A theoretical review towards a unifying evidence-based definition. Sleep Med Rev 2019;44:70-82. http://dx.doi.org/10.1016/j.smrv.2018.12.007

11. Marzano C, Ferrara M, Sforza E, De Gennaro L. Quantitative electroencephalogram (EEG) in insomnia: a new window on pathophysiological mechanisms. Curr Pharm Des 2008;14(32):3446-55. http://dx.doi.org/10.2174/138161208786549326

12. Riemann D, Spiegelhalder K, Feige B, et al. The hyperarousal model of insomnia: A review of the concept and its evidence. Sleep Med Rev 2010;14(1):19-31. http://dx.doi.org/10.1016/j.smrv.2009.04.002.

13. Perlis ML, Smith MT, Andrews PJ, Orff H, Giles DE. Beta/Gamma EEG activity in patients with primary and secondary insomnia and good sleeper controls. Sleep 2001;24(1):110-7. http://dx.doi.org/10.1093/sleep/24.1.110 
14. Spiegelhalder K, Regen W, Feige B, et al. Increased EEG sigma and beta power during NREM sleep in primary insomnia. Biol Psychol 2012;91(3):329-33. http://dx.doi.org/10.1016/j.biopsycho.2012.08.009

15. Kwan Y, Baek C, Chung S, Kim TH, Choi S. Resting-state quantitative EEG characteristics of insomniac patients with depression. Int $\mathrm{J}$ Psychopyhsiol 2018;124:26-32. http://dx.doi.org/10.1016/j.ijpsycho.2018.01.005

16. Riedner BA, Goldstein MR, Plante DT, et al. Regional Patterns of Elevated Alpha and High-Frequency Electroencephalographic Activity during Nonrapid Eye Movement Sleep in Chronic Insomnia: A Pilot Study. Sleep 2016;39(4):801-12. http://dx.doi.org/10.5665/sleep.5632

17. Colombo MA, Ramautar JR, Wei Y, et al. Wake High-Density Electroencephalographic Spatiospectral Signatures of Insomnia. Sleep 2016;39(5):1015-27. http://dx.doi.org/10.5665/sleep.5744

18. LAMARCHE CH, OGILVIE RD. Electrophysiological changes during the sleep onset period of psychophysiological insomniacs, psychiatric insomniacs, and normal sleepers. Sleep (New York, N.Y.) 1997;20(9):724-33.

19. Krystal AD, Edinger JD, Wohlgemuth WK, Marsh GR. NREM sleep EEG frequency spectral correlates of sleep complaints in primary insomnia subtypes. Sleep 2002;25(6):630-40.

20. St-Jean G, Turcotte I, Pérusse AD, Bastien CH. REM and NREM power spectral analysis on two consecutive nights in psychophysiological and paradoxical insomnia $\begin{array}{lllll}\text { sufferers. } & \text { Int } & \mathrm{J} & \text { Psychopyhsiol 2013;89(2):181-94. }\end{array}$ http://dx.doi.org/10.1016/j.ijpsycho.2013.06.004

21. Lecci S, Cataldi J, Betta M, Bernardi G, Heinzer R, Siclari F. EEG changes associated with subjective under- and overestimation of sleep duration. Sleep (New York, N.Y.) 2020;43(11).http://dx.doi.org/10.1093/sleep/zsaa094 
22. Chellappa SL, Munch M, Knoblauch V, Cajochen C. Age effects on spectral electroencephalogram activity prior to dream recall. J Sleep Res 2012;21(3):247-56. http://dx.doi.org/10.1111/j.1365-2869.2011.00947.x

23. Svetnik V, Snyder ES, Ma J, Tao P, Lines C, Herring WJ. EEG spectral analysis of NREM sleep in a large sample of patients with insomnia and good sleepers: effects of age, sex and part of the night. J Sleep Res 2017;26(1):92-104. http://dx.doi.org/ $\underline{10.1111 / \text { jsr. } 12448}$

24. Dijk D, Duffy JF, Riel E, Shanahan TL, Czeisler CA. Ageing and the circadian and homeostatic regulation of human sleep during forced desynchrony of rest, melatonin and temperature rhythms. The Journal of physiology 1999;516(2):611-27. http://dx.doi.org/10.1111/j.1469-7793.1999.0611v.x

25. Liu XC, Tang MQ, Hu L, et al. Reliability and validity of the Pitts-burgh Sleep Quality Index-Chinese Version. Chin J Psychiatry 1996; 29: 103-7.

26. Bonicatto S, Dew MA, Soria JJ, Seghezzo ME. Validity and reliability of Symptom Checklist '90 (SCL90) in an Argentine population sample. Soc Psychiatry Psychiatr Epidemiol 1997;32(6):332-8. http://dx.doi.org/10.1007/BF00805438

27. Berry RB, R B, E GC, M HS, M LR. The AASM Manual for the Scoring of Sleep and Associated Events. Version 2.5. Darien, IL, USA: American Academy of Sleep Medicine, 2018.

28. BRUNNER D, VASKO R, DETKA C, MONAHAN J, REYNOLDS III C, KUPFER D. Muscle artifacts in the sleep EEG: Automated detection and effect on all-night EEG power spectra. J Sleep Res 1996;5(3):155-64. http://dx.doi.org/10.1046/j.1365-2869.1996.00009.x 
29. Merica H, Gaillard JM. The EEG of the sleep onset period in insomnia: a discriminant analysis. Physiol Behav 1992;52(2):199. http://dx.doi.org/10.1016/0031$\underline{9384(92) 90258-4}$

30. Cervena K, Espa F, Perogamvros L, Perrig S, Merica H, Ibanez V. Spectral analysis of the sleep onset period in primary insomnia. Clin Neurophysiol 2014;125(5):979-87. http://dx.doi.org/10.1016/j.clinph.2013.10.010

31. Ferri R, Cosentino FII, Manconi M, Rundo F, Bruni O, Zucconi M. Increased Electroencephalographic High Frequencies during the Sleep Onset Period in Patients with Restless Legs Syndrome. Sleep 2014;37(8):1375-81. http://dx.doi.org/10.5665/sleep.3934

32. Maes J, Verbraecken J, Willemen M, et al. Sleep misperception, EEG characteristics and Autonomic Nervous System activity in primary insomnia: A retrospective study on polysomnographic data. Int J Psychophysiol 2014;91(3):163-71. http://dx.doi.org/10.1016/j.ijpsycho.2013.10.012 
Table 1 Demographic characteristics of all participants

\begin{tabular}{|c|c|c|c|c|}
\hline Variable & $\mathrm{GS}(\mathrm{n}=10)$ & $\begin{array}{c}\text { IWHM ( } \mathrm{n}= \\
18)\end{array}$ & $\begin{array}{c}\text { IWLM ( } \mathrm{n}= \\
19)\end{array}$ & Statistics \\
\hline Age, years & $26.90 \pm 2.89$ & $31.39 \pm 6.11$ & $28.95 \pm 7.04$ & $\chi^{2}=3.378, p=0.185$ \\
\hline $\operatorname{Sex}(\mathrm{F} / \mathrm{M})$ & $5 / 5$ & $9 / 9$ & $16 / 3$ & $\chi^{2}=5.616, p=0.060$ \\
\hline Race & & & & $\chi^{2}=0.000, p=1.000$ \\
\hline Han & 10 & 18 & 19 & \\
\hline Non-Han & 0 & 0 & 0 & \\
\hline $\begin{array}{l}\text { Place of } \\
\text { residence }\end{array}$ & & & & $\chi^{2}=1.233, p=0.540$ \\
\hline Downtown & 9 & 13 & 15 & \\
\hline Suburb & 1 & 4 & 3 & \\
\hline Village & 0 & 1 & 1 & \\
\hline Marriage & & & & $\chi^{2}=2.798, p=0.247$ \\
\hline Unmarried & 7 & 7 & 10 & \\
\hline Married & 3 & 10 & 9 & \\
\hline $\begin{array}{c}\text { Bereavement/ } \\
\text { divorce }\end{array}$ & 0 & 1 & 0 & \\
\hline Family history & & & & \\
\hline $\begin{array}{l}\text { of insomnia } \\
(\mathrm{Y} / \mathrm{N})\end{array}$ & $1 / 9$ & $3 / 15$ & $1 / 18$ & $\chi^{2}=1.243, p=0.537$ \\
\hline Family history & & & & \\
\hline $\begin{array}{l}\text { of psychosis } \\
(\mathrm{Y} / \mathrm{N})\end{array}$ & $0 / 10$ & $0 / 18$ & $1 / 18$ & $\chi^{2}=1.474, p=0.479$ \\
\hline
\end{tabular}


Data are mean \pm standard deviation $(\mathrm{SD})$ or $\mathrm{n}(\%)$ values.

GS, good sleeper; IWLM, insomnias with a low mismatch; IWHM, insomnias with a high mismatch

Table 2- PSQI scores, SCL90scores, and PSGcharacteristics of all participants.

\begin{tabular}{ccccc}
\hline Variable & GS $(n=10)$ & IWHM $(n=$ & $\operatorname{IWLM}(\mathrm{n}=$ & Statistics \\
& $18)$ & $19)$ &
\end{tabular}

$\begin{array}{ccccc}\text { PSQI total } & 3.80 \pm 2.39 & 13.17 \pm 3.65^{\mathrm{a}} & 11.29+4.44^{\mathrm{a}} & \chi^{2}=18.882, p=0.000 \\ \text { score } & & & & \\ \text { SCL-90 total } & & & & \\ \text { score } & 115.90 \pm 16.63 & 185.44 \pm 51.41^{\mathrm{a}} & 155.00 \pm 39.57^{\mathrm{a}} & \chi^{2}=16.037, p=0.000 \\ \text { TST (min) } & 394.25 \pm 45.25 & 415.06 \pm 40.20 & 381.95 \pm 39.85 & \mathrm{~F}=3.026, p=0.059 \\ \text { SPT(min) } & 418.60 \pm 47.39 & 459.03 \pm 42.65 & 431.84 \pm 49.77 & \mathrm{~F}=2.837 p=0.069 \\ \text { SE, \% } & 89.27 \pm 3.92 & 87.84 \pm 7.00 & 86.24 \pm 8.39 & \mathrm{~F}=0.626, p=0.540 \\ \text { SOL (min) } & 13.75 \pm 9.01 & 10.58+10.37 & 8.24 \pm 6.84 & \chi^{2}=2.697, p=0.260 \\ \text { \%NREM } & & & & \\ \text { stage1 } & 4.70 \pm 1.70 & 6.00 \pm 3.03 & 5.42 \pm 2.83 & \chi^{2}=1.158, p=0.560 \\ \text { \%NREM } & & & & \\ \text { \% } & & & & \\ \text { \% Stage2 } & 59.40 \pm 8.51 & 61.17 \pm 8.61 & 62.16 \pm 7.63 & \mathrm{~F}=0.371, p=0.692 \\ \text { \% SW } & 13.60 \pm 4.79 & 10.06 \pm 6.49 & 12.37 \pm 4.82 & \mathrm{~F}=1.528, p=0.228 \\ & 21.90 \pm 6.61 & 22.61 \pm 3.27 & 19.31 \pm 5.14 & \mathrm{~F}=2.259, p=0.116\end{array}$

Number of

$23.50 \pm 6.38 \quad 23.56 \pm 10.27 \quad 20.68 \pm 10.52 \quad \chi^{2}=2.147, p=0.342$

awakings

Number of

$21.20+25.47 \quad 55.67 \pm 75.98 \quad 92.11 \pm 157.88 \quad \chi^{2}=3.730, p=0.155$

arousal 


\begin{tabular}{lcccc}
\hline Arousal index & $3.29 \pm 3.94$ & $8.12 \pm 10.95$ & $14.17 \pm 23.99$ & $\chi^{2}=3.611, p=0.164$ \\
Number of & & & \\
arousal of & $20.10 \pm 25.74$ & $53.50 \pm 76.03$ & $90.00 \pm 158.10$ & $\chi^{2}=3.233, p=0.199$ \\
NREM & & & & \\
Number of & & & \\
arousal of & $1.10 \pm 1.60$ & $2.17 \pm 2.75$ & $2.11 \pm 2.73$ & $\chi^{2}=1.339, p=0.512$ \\
REM & & & &
\end{tabular}

Data are shown mean \pm standard deviation (SD) or $\mathrm{n}(\%)$.

GS, good sleeper; IWLM, insomnias with a low mismatch; IWHM, insomnias with a high mismatch

PSQI, Pittsburgh Sleep Quality Index; TST, total sleep time; SPT, sleep period time; SE, sleep efficiency; SOL, sleep onset latency; NREM: non-rapid eye movement; SWS, slow wave sleep; REM, rapid eye movement ${ }^{\mathrm{a}} p<0.05$ versus GS.

Table 3- Comparison of absolute EEG spectral power among experimental groups $\left(\mu v^{2}\right)$ $\operatorname{IWHM}(\mathrm{n}=\operatorname{IWLM}(\mathrm{n}=$ Variable $\quad$ GS $(n=10)$

19) Statistics

\begin{tabular}{ccccc}
\hline Frontal derivation & & & & \\
Delta $(1-4 \mathrm{~Hz})$ & $46.47 \pm 21.05$ & $37.61 \pm 15.30$ & $46.65 \pm 25.95$ & $\mathrm{~F}=0.981, p=0.383$ \\
Theta $(4-8 \mathrm{~Hz}$ & $3.23 \pm 1.32$ & $6.54 \pm 10.21$ & $4.89 \pm 2.77$ & $\chi^{2}=3.481, p=0.175$ \\
Alpha (8-12 Hz) & $2.14 \pm 1.09$ & $2.47 \pm 0.91$ & $3.55 \pm 2.54$ & $\chi^{2}=2.693, p=0.260$ \\
Beta (15-20 Hz) & $1.18 \pm 0.51$ & $1.82 \pm 0.85$ & $2.23 \pm 1.35^{\mathrm{a}}$ & $\chi^{2}=6.454, p=0.040$ \\
Beta /Delta & $3.19 \pm 2.45$ & $5.68 \pm 4.42^{\mathrm{a}}$ & $9.34 \pm 20.25^{\mathrm{a}}$ & $\chi^{2}=7.783, p=0.020$ \\
Beta/Theta & $38.15 \pm 11.93$ & $56.80 \pm 37.98$ & $49.26 \pm 23.47$ & $\chi^{2}=2.880, p=0.237$ \\
Alpha/ Delta & $5.32 \pm 3.26$ & $7.66 \pm 4.93$ & $20.75 \pm 57.75$ & $\chi^{2}=3.086, p=0.214$ \\
\hline
\end{tabular}




\begin{tabular}{lllll}
\hline Alpha/Theta & $64.00 \pm 16.43$ & $76.60 \pm 41.44$ & $74.33 \pm 30.73$ & $\mathrm{~F}=0.487, p=0.618$
\end{tabular}

Central derivation

$\begin{array}{ccccc}\text { Delta }(1-4 \mathrm{~Hz}) & 37.99 \pm 12.36 & 33.89 \pm 19.01 & 46.48 \pm 20.94 & \mathrm{~F}=2.154, p=0.128 \\ \text { Theta }(4-8 \mathrm{~Hz} & 4.22 \pm 1.23 & 4.22 \pm 1.53 & 6.01 \pm 3.42 & \chi^{2}=2.872, p=0.238 \\ \text { Alpha }(8-12 \mathrm{~Hz}) & 2.49 \pm 1.06 & 2.84 \pm 1.10 & 3.88 \pm 3.48 & \chi^{2}=0.710, p=0.701 \\ \text { Beta }(15-20 \mathrm{~Hz}) & 1.76 \pm 0.48 & 2.59 \pm 1.07^{\mathrm{a}} & 2.82 \pm 1.22^{\mathrm{a}} & \mathrm{F}=3.480, p=0.040 \\ \text { Beta/Delta } & 4.98 \pm 1.84 & 13.06 \pm 17.26^{\mathrm{a}} & 6.57 \pm 2.69 & \chi^{2}=6.861, p=0.032 \\ \text { Beta/Theta } & 44.59 \pm 16.04 & 65.91 \pm 35.76 & 55.74 \pm 31.10 & \chi^{2}=4.860, p=0.088 \\ \text { Alpha/Delta } & 6.87 \pm 2.90 & 14.20 \pm 20.91 & 8.76 \pm 8.42 & \chi^{2}=2.632, p=0.268 \\ \text { Alpha/Theta } & 57.29 \pm 13.21 & 70.71 \pm 29.13 & 63.31 \pm 32.98 & \chi^{2}=1.774, p=0.412\end{array}$

Data are shown as mean \pm standard deviation (SD).

GS, good sleeper; IWLM, insomnias with a low mismatch; IWHM, insomnias with a high mismatch; ${ }^{\mathrm{a}} p<0.05$ versus GS.

Table 4- Comparison of relative EEG spectral power among experimental groups

\begin{tabular}{lcccc}
\hline Variable & GS $(\mathrm{n}=10)$ & IWHM $(\mathrm{n}=$ & IWLM $(\mathrm{n}=$ & Statistics \\
& & $18)$ & $19)$ & \\
\hline Frontal derivation & & & & \\
Delta (1-4 Hz) & $86.27 \pm 6.92$ & $78.24 \pm 11.88$ & $78.30 \pm 16.78$ & $\chi^{2}=3.820, p=0.148$ \\
Theta (4-8 Hz & $6.73 \pm 3.24$ & $11.62 \pm 12.53$ & $10.14 \pm 8.61$ & $\chi^{2}=5.243, p=0.073$ \\
Alpha(8-12 Hz) & $4.39 \pm 2.33$ & $5.83 \pm 3.14$ & $7.35 \pm 6.67$ & $\chi^{2}=2.805, p=0.246$ \\
Beta(15-20 Hz) & $2.61+1.71$ & $4.30 \pm 2.85$ & $4 . .21 \pm 2.14^{\mathrm{a}}$ & $\chi^{2}=7.153, p=0.028$ \\
Beta /Delta & $3.19 \pm 2.45$ & $5.68 \pm 4.42^{\mathrm{a}}$ & $9.34 \pm 20.25^{\mathrm{a}}$ & $\chi^{2}=7.783, p=0.020$ \\
Beta/Theta & $38.15 \pm 11.93$ & $56.80 \pm 37.98$ & $49.26 \pm 23.47$ & $\chi^{2}=2.880, p=0.237$ \\
\hline
\end{tabular}




\begin{tabular}{lcccc}
\hline Alpha/Delta & $5.32 \pm 3.26$ & $7.66 \pm 4.93$ & $20.75 \pm 57.75$ & $\chi^{2}=0.214, p=0.214$ \\
Alpha/Theta & $64.00 \pm 16.42$ & $76.60 \pm 41.44$ & $74.32 \pm 30.73$ & $\mathrm{~F}=0.487, p=0.618$ \\
Central derivation & & & & \\
Delta (1-4 Hz) & $81.30 \pm 5.14$ & $73.56 \pm 14.76$ & $78.46 \pm 7.26$ & $\chi^{2}=3.587, p=0.166$ \\
Theta (4-8 Hz & $9.26 \pm 2.43$ & $11.47 \pm 7.14$ & $10.18 \pm 3.25$ & $\chi^{2}=0.342, p=0.843$ \\
Alpha(8-12 Hz) & $5.46 \pm 2.11$ & $7.70 \pm 4.44$ & $6.34 \pm 4.38$ & $\chi^{2}=2.483, p=0.289$ \\
Beta(15-20 Hz) & $3.99 \pm 1.30$ & $7.28 \pm 4.62^{\mathrm{a}}$ & $5.02 \pm 1.52$ & $\chi^{2}=6.963, p=0.031$ \\
Beta /Delta & $4.98 \pm 1.84$ & $13.06 \pm 17.26^{\mathrm{a}}$ & $6.47 \pm 4.37$ & $\chi^{2}=6.861, p=0.032$ \\
Beta/ Theta & $44.59 \pm 16.04$ & $65.91 \pm 35.76$ & $55.73 \pm 3.11$ & $\chi^{2}=4.860, p=0.088$ \\
Alpha/ Delta & $6.87 \pm 2.90$ & $14.20 \pm 20.91$ & $8.76 \pm 8.42$ & $\chi^{2}=2.632, p=0.268$ \\
Alpha/ Theta & $57.29 \pm 13.21$ & $70.71 \pm 29.13$ & $63.31 \pm 32.98$ & $\chi^{2}=1.774, p=0.412$ \\
\hline
\end{tabular}

Data are shown as mean \pm standard deviation (SD).

GS, good sleeper; IWLM, insomnias with a low mismatch; IWHM, insomnias with a high mismatch; ${ }^{\mathrm{a}} \mathrm{p}<0.05$ versus GS.

Table 5- Correlation between absolute EEG spectral power andSOD of TST

\begin{tabular}{ccc}
\hline Variables & \multicolumn{3}{c}{ SOD of TST } \\
\cline { 2 - 3 } & $\mathrm{r}$ & $p$ \\
\hline Frontal derivation & 0.157 & 0.290 \\
Delta (1-4 Hz) & 0.114 & 0.447 \\
Theta (4-8 Hz & 0.024 & 0.871 \\
Alpha (8-12 Hz) & 0.074 & 0.622 \\
Beta (15-20 Hz) & 0.139 & 0.353 \\
Beta / Delta & 0.217 & 0.142 \\
Beta/ Theta & &
\end{tabular}




\begin{tabular}{ccc}
\hline Alpha/ Delta & 0.102 & 0.495 \\
Alpha/ Theta & 0.179 & 0.229 \\
Central derivation & 0.114 & 0.445 \\
Delta (1-4 Hz) & 0.029 & 0.539 \\
Theta (4-8 Hz & 0.047 & 0.752 \\
Alpha (8-12 Hz) & 0.196 & 0.187 \\
Beta (15-20 Hz) & 0.314 & 0.032 \\
Beta / Delta & 0.190 & 0.200 \\
Beta/ Theta & 0.256 & 0.083 \\
Alpha/ Delta & 0.264 & 0.073 \\
Alpha/ Theta & &
\end{tabular}

SOD, Subjective-objective sleep discrepancy; TST, total sleep time.

Table 6- Correlation between relative EEG spectral power and SOD of TST

\begin{tabular}{ccc}
\hline Variables & \multicolumn{2}{c}{ SOD of TST } \\
\cline { 2 - 3 } & $\mathrm{r}$ & \\
\hline Frontal derivation & 0.202 & 0.172 \\
Delta (1-4 Hz) & 0.153 & 0.305 \\
Theta (4-8 Hz & 0.141 & 0.343 \\
Alpha (8-12 Hz) & 0.241 & 0.103 \\
Beta (15-20 Hz) & 0.139 & 0.353 \\
Beta / Delta & 0.217 & 0.142 \\
Beta/ Theta & 0.102 & 0.495 \\
Alpha/ Delta & 0.179 & 0.229 \\
Alpha/ Theta & &
\end{tabular}


Central derivation

$\begin{array}{ccc}\text { Delta }(1-4 \mathrm{~Hz}) & -0.289 & 0.049 \\ \text { Theta }(4-8 \mathrm{~Hz} & 0.156 & 0.294 \\ \text { Alpha }(8-12 \mathrm{~Hz}) & 0.269 & 0.067 \\ \text { Beta }(15-20 \mathrm{~Hz}) & 0.373 & 0.010 \\ \text { Beta / Delta } & 0.314 & 0.032 \\ \text { Beta/ Theta } & 0.190 & 0.200 \\ \text { Alpha/ Delta } & 0.256 & 0.083 \\ \text { Alpha/ Theta } & 0.264 & 0.073\end{array}$

SOD, Subjective-objective sleep discrepancy; TST, total sleep time 